\title{
Optimizing CASCADE Data Aggregation for VANETs
}

\author{
Khaled Ibrahim and Michele C. Weigle \\ Department of Computer Science, Old Dominion University \\ Norfolk, VA 23529 \\ E-mail: $\{$ ibrah_k, mweigle\}@cs.odu.edu
}

\begin{abstract}
We present an analysis of the CASCADE (Cluster-based Accurate Syntactic Compression of Aggregated Data in VANETS) data aggregation technique. CASCADE organizes known vehicles into clusters, the size of which determines both the frame size used to distribute aggregated data and the distance ahead that vehicles are aware of (local view). In this paper, we determine the optimal cluster size to balance the trade-off between local view length and expected frame size.
\end{abstract}

\section{Introduction}

Vehicular Ad-hoc Networks (VANETs) have been proposed to provide drivers with advance notification of traffic congestion using wireless communication. Most applications using VANETs require vehicles to exchange position updates several times a second, which can cause contention on the wireless channel. To propagate traffic information to vehicles outside of the original vehicle's transmission range, VANET messages are often re-broadcasted multiple times. The more vehicles participating in the VANET, the more messages are sent, and the higher the probability of wireless collisions.

Recently, data aggregation in VANETs has received much attention to reduce the number of messages that are sent [4-6]. CASCADE (Cluster-based Accurate Syntactic Compression of Aggregated Data in VANETs) [2,3] is a data aggregation technique, proposed to reduce also, the number of messages that are sent while ensuring that accurate information is propagated. CASCADE organizes vehicles into clusters, and the amount of information that a vehicle has about its surroundings depends upon the defined cluster size. As shown in Figure 1, the original CASCADE proposal had a $4 \mathrm{~m}$ wide by $62 \mathrm{~m}$ long cluster size, which provided a local view of $1.9 \mathrm{~km}$, but at the expense of having a large frame size. In this paper, we analyze the trade-off between frame size and local view length to determine the optimal cluster dimensions for CASCADE.

\section{Overview of CASCADE}

In CASCADE, vehicles pass information about surrounding vehicles to following vehicles, giving them advance notification of upcoming traffic conditions. Here we provide a brief overview of the CASCADE system.

\subsection{Primary Frames}

Each vehicle broadcasts a primary frame every 300$400 \mathrm{~ms}$. This primary frame consists of position information about the vehicle, including location, speed, acceleration, and heading. When a vehicle receives a primary frame, it extracts this information and stores it in a primary record. A vehicle's local view is made up of primary records representing vehicles a certain distance ahead. The distance covered by the local view depends upon the number of vehicle records that can fit in a single IEEE 802.11 frame (2312 bytes). CASCADE compresses and aggregates primary records so that many more vehicles can be represented in a single frame than if no compression was performed. If the length of the local view is greater than the transmission range, about $300 \mathrm{~m}$, the primary frames are rebroadcast using probabilistic-IVG (p-IVG) [3], an enhancement of Inter-Vehicle Geocast (IVG) [1] that considers the density of surrounding vehicles in determining which vehicle will re-broadcast the frame.

\section{$2.2 \quad$ Aggregated Frames}

Every four seconds, a vehicle will compress, aggregate, and broadcast its local view as an aggregated frame. To achieve compression, each vehicle in CASCADE divides the road ahead into fixed-sized clusters. Then, each vehicle is represented by the difference between it and the cluster center. The original cluster size in CASCADE was $4 \mathrm{~m}$ wide (width of a lane) x $62 \mathrm{~m}$ long. Our contribution in this paper 

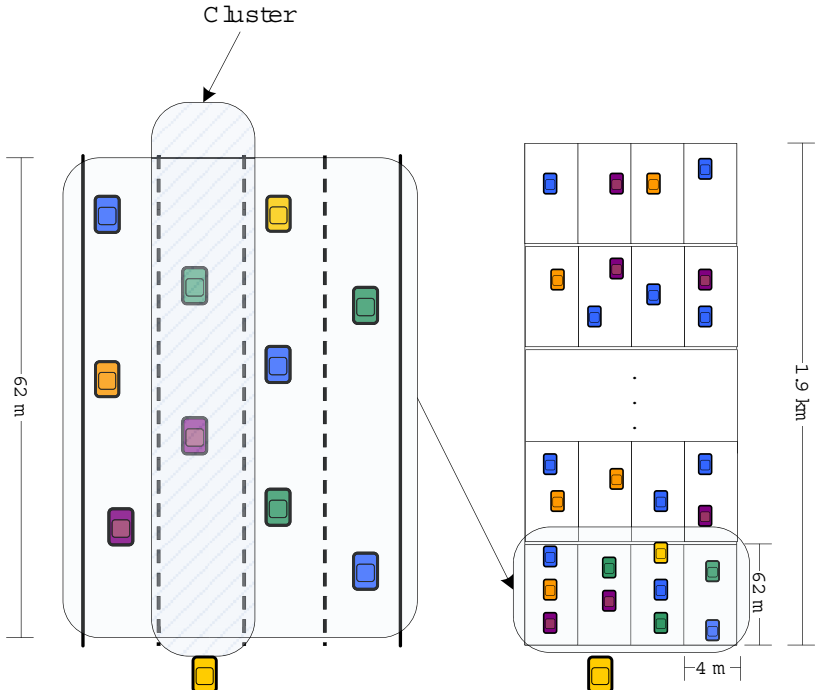

Figure 1. Vehicle's Local View, Divided into $4 \mathrm{~m} \times 62 \mathrm{~m}$ Clusters

is to optimize the cluster size to minimize the aggregated frame size while maximizing the local view length.

When a vehicle receives a primary frame describing a vehicle, that vehicle is grouped into its appropriate cluster, based on its heading and distance from the receiving vehicle. When the receiving vehicle performs compression and aggregation, the location and speed of each vehicle relative to its cluster is represented in a compact record (CR). The position of each vehicle is translated into $\{X, Y\}$ coordinates (in integer meters) with the local view origin as point $(0,0)$. Each CR consists of the following:

- $\Delta X$ - difference between the vehicle's $X$ coordinate position and the $X$ coordinate for the center of its cluster in sign-magnitude representation

- $\Delta Y$ - the difference between the vehicle's $Y$ coordinate position and the $Y$ coordinate for the center of its cluster in sign-magnitude representation

- $\Delta S$ (5 bits) - the difference between vehicle's speed and the median speed of the vehicles in the cluster in sign-magnitude representation

- Speed Indicator (SI) Flag (2 bits) - indicates if the vehicle's speed is within the acceptable range for the cluster (within $[-15 \mathrm{~m} / \mathrm{s}, 15 \mathrm{~m} / \mathrm{s}]$ of the median speed)

In this paper, we investigate the optimal cluster size, which will affect the size of $\Delta X$ and $\Delta Y$.

The range of acceptable values for $\Delta S$ is $[-15 \mathrm{~m} / \mathrm{s}$, $15 \mathrm{~m} / \mathrm{s}$ ]. If the difference is outside of this range, then the $\Delta S$ field will be omitted, and the SI Flag will be set. The $S I$ Flag can take one of three possible values $\{00,01,10\}$ :
- $00-\Delta S$ can be represented in the allowed range [min $\Delta S, \max \Delta S]$

- $01-\Delta S>\max \Delta S$, the vehicle is a speeder

- $10-\Delta S<\min \Delta S$, the vehicle is a lagger

Once the CRs for all vehicles in the local view have been created, an aggregated cluster record (ACR) is formed for each cluster. Each aggregated cluster record contains the following fields:

- cluster flag (1 bit) - indicates if the cluster contains any vehicles

- cluster median speed (8 bits) - the median speed of the vehicles in the cluster in meters/second

- number of vehicles - the number of vehicles in the cluster

- compact data records - concatenation of the CDRs in the cluster

If a cluster contains no vehicles, it is represented by a single bit (the cluster flag set to 0). Since the number of vehicles in a cluster depends upon the cluster size, we will discuss the optimal sizes for the last two fields later.

Once the ACRs are constructed, they are concatenated into a single frame and sent via broadcast. The aggregated frame (Figure 2) contains the following fields:

- type (1 bit) - primary or aggregated frame

- timestamp ( 8 bytes)

- sender's location (16 bytes) - latitude/longitude

- aggregating vehicle's X-coordinate - meters from the vehicle's local view origin

- aggregating vehicle's location (16 bytes) - latitude/longitude

- aggregated cluster records - concatenation of all ACRs in the local view, arranged according to their position in the local view starting with the bottom-left cluster

- digital signature (28 bytes)

- certificate (56 bytes)

By using differences in constructing the compact record, we achieve a compression ratio of at least $88 \%$. The primary data for each vehicle (location and speed) is represented in 136 bits (17 bytes) while the compact record for each vehicle is represented in at most 16 bits. The compression ratio is even higher if the $\Delta S$ field is omitted, as in the case of speeders and laggers. 


\begin{tabular}{|c|c|c|c|c|c|c|c|c|c|}
\hline \multirow{2}{*}{$\begin{array}{l}\text { Frame } \\
\text { Type } \\
\text { (1 Bit) }\end{array}$} & \multirow{2}{*}{$\begin{array}{c}\text { Timestamp } \\
\text { (8 Bytes) }\end{array}$} & \multicolumn{2}{|c|}{ The Sender Location } & \multicolumn{3}{|c|}{ The View's Base Point } & \multirow{2}{*}{$\begin{array}{c}\text { Data } \\
\text { Section } \\
\text { (Variable) }\end{array}$} & \multirow{2}{*}{$\begin{array}{l}\text { Signature } \\
\text { (28 Bytes) }\end{array}$} & \multirow{2}{*}{$\begin{array}{l}\text { Certificate } \\
\text { (56 Bytes) }\end{array}$} \\
\hline & & $\begin{array}{l}\text { (Latitude) } \\
\text { (8 Bytes) }\end{array}$ & $\begin{array}{c}\text { (Longitude) } \\
\text { (8 Bytes) }\end{array}$ & $\begin{array}{c}\text { X-coordinate } \\
\text { (Variable) }\end{array}$ & $\begin{array}{c}\text { (Latitude) } \\
\text { (8 Bytes) }\end{array}$ & $\begin{array}{c}\text { (Longitude) } \\
\text { (8 Bytes) }\end{array}$ & & & \\
\hline
\end{tabular}

Figure 2. Aggregated Frame

\section{Determining Optimal Cluster Size}

In previous work, it was assumed that each CASCADE cluster was $4 \mathrm{~m}$ wide and $62 \mathrm{~m}$ long, resulting in a local view size of $1.9 \mathrm{~km}$. We investigate and determine the optimal cluster size in terms of the size of the aggregated frames created and the length of the local view that results. As primary frames contain information about only a single vehicle, they are not affected by the cluster size. In determining the optimal cluster size, we strive to find an appropriate trade-off that will minimize the aggregated frame size and maximize the local view length.

\subsection{Aggregated Frame Size}

The aggregated frame contains the compressed records of all vehicles in the aggregating vehicle's local view and is useful for vehicles behind the aggregating vehicle. The data section of the aggregated frame consists of the concatenation of all of the aggregated cluster records (ACRs) in the local view. Each ACR represents a single cluster and consists of a header and the compact records (CRs) of all vehicles in the cluster.

We will follow a bottom-up approach in investigating the relationship between the aggregated frame size and the cluster size. We first discuss of the size of a CR (describes one vehicle), then the size of an ACR (describes all of the vehicles in a single cluster), and finally the size of the aggregated frame.

\subsubsection{Compact Record (CR)}

The size of a CR depends upon the size of the fields $\Delta X$, $\Delta Y, \Delta S$, and SIFlag. Neither the size of $\Delta S$ (5 bits) nor the SIFlag (2 bits) depend upon the cluster length, so we focus on the size of $\Delta X$ and $\Delta Y$.

The values $\Delta X$ and $\Delta Y$ for each vehicle are calculated with respect to the cluster center, so the number of bits allocated to each depends on the cluster width $W_{C}$ and cluster length $L_{C}$ as shown in Equations 1 and 2, respectively.

$$
\begin{gathered}
\Delta X . \text { size }=\left\lceil\log _{2}\left(\frac{W_{C}}{2}+1\right)\right\rceil+1 \\
\Delta Y . \text { size }=\left\lceil\log _{2}\left(\frac{L_{C}}{2}+1\right)\right\rceil+1
\end{gathered}
$$

Recall that $\Delta X$ and $\Delta Y$ are expressed using signmagnitude representation, so an extra bit is added to hold the sign. As the cluster width and length increase, the number of bits allocated for $\Delta X$ and $\Delta Y$ increases, respectively. Equation 3 shows the number of bits needed to represent a $\mathrm{CR}$ as a function of the cluster width and cluster length.

$$
\text { CR.size }=\left\lceil\log _{2}\left(\frac{W_{C}}{2}+1\right)\right\rceil+\left\lceil\log _{2}\left(\frac{L_{C}}{2}+1\right)\right\rceil+9
$$

\subsubsection{Aggregated Cluster Record (ACR)}

The ACR represents all of the vehicles in a cluster. The maximum number of vehicles $V_{\max }$ in a cluster depends on the size of the cluster, the size of a single lane, and the average size of a vehicle. We assume that the width of one lane is $4 \mathrm{~m}$ and that the average vehicle length is $5 \mathrm{~m}$, so $V_{\max }$ can be expressed as in Equation 4.

$$
V_{\max }=\left\lfloor\frac{L_{C}}{5}\right\rfloor *\left\lfloor\frac{W_{C}}{4}\right\rfloor
$$

The size of the ACR can be broken up into the size of the header part $A C R_{-} H_{e a d e r . s i z e}$ and the size of the data part ACR_Data.size.

The header section in the ACR consists of the cluster flag, cluster median speed, and number of vehicles. The sizes of both the cluster flag (1 bit) and the cluster median speed ( 8 bits) are constant, so they do not depend on the cluster dimensions. The number of bits allocated for the number of vehicles field $V_{\text {count }}$ depends on the cluster size dimensions and is shown in Equation 5.

$$
V_{\text {count. }} \text { size }=\left\lceil\log _{2}\left(V_{\max }+1\right)\right\rceil
$$

Thus, the size of the ACR header can be represented by Equation 6.

$$
A C R \_H e a d e r . s i z e=\left\lceil\log _{2}\left(V_{\max }+1\right)\right\rceil+9
$$

The data section of the ACR contains the CRs of all vehicles in the cluster. The maximum size of the data section is bounded by the size of the CR multiplied by $V_{\max }$ (Equation 7), while the actual size depends upon the number of vehicles in the cluster (Equation 8).

$$
\begin{gathered}
M a x \_A C R \_D a t a . s i z e=V_{\max } * C R . s i z e \\
A C R \_D a t a . s i z e=V_{\text {count }} * C R . s i z e
\end{gathered}
$$




\subsubsection{Aggregated Frame (AF)}

Now that we have equations for the size of the CR and ACR, we can look at how the size of the aggregated frame is affected by the cluster size. The fields in the aggregated frame are shown in Figure 2. There are only two fields that are variable in size: the $X$-coordinate of the aggregator and the concatenation of the ACRs, labeled Data Section in the figure. The remaining fields contribute 993 bits to the size of the aggregated frame. The total size of the AF in bits can be represented by Equation 9.

$$
\text { AF.size }=\text { X_Coord.size }+ \text { AF_Data.size }+993
$$

The $X$-coordinate of the aggregator is the position of the aggregator measured as the number of meters from the left edge of the leftmost lane. The number of bits needed to represent this (Equation 10) depends upon the size of each lane (we assume $4 \mathrm{~m}$ ) and the number of lanes on the roadway $C_{L}$.

$$
X_{-} \text {Coord.size }=\left\lceil\log _{2}\left(4 * C_{L}\right)+1\right\rceil
$$

The Data Section consists of the concatenation of all of the ACRs in the local view. Each ACR represents a single cluster. If there are no vehicles in the cluster, the ACR is represented by a single bit. We show the size AF_Data.size in Equation 11, where ACR.count is the number of ACRs that can fit in the frame and $v_{i}$ is the number of vehicles in cluster $i$.

$$
\text { AF_Data.size }=\sum_{i=1}^{A C R . c o u n t} \begin{cases}A C R \_D a t a_{i} . \text { size } & \text { if } v_{i}>0 \\ 1 & \text { if } v_{i}=0\end{cases}
$$

The maximum number of ACRs (ACR.count) that can be in the frame depends upon the maximum frame size (MAC_Frame.size), the size of the frame without the Data Section (AF_nodata.size), and the size of each ACR (ACR.size), as shown in Equation 12.

$$
A C R . c o u n t=\left\lfloor\frac{M A C \_F r a m e . s i z e-A F \_n o d a t a . s i z e}{A C R . s i z e}\right\rfloor
$$

\subsection{Local View Length Analysis}

The goal of our analysis is to find a cluster size that will minimize the aggregated frame size while maximizing the local view length. The length of the local view, which determines how much information about vehicles ahead can be passed to vehicles behind, is dependent upon the size of the aggregated frame. An important constraint on frame size is the maximum IEEE 802.11 frame size of 2312 bytes. The

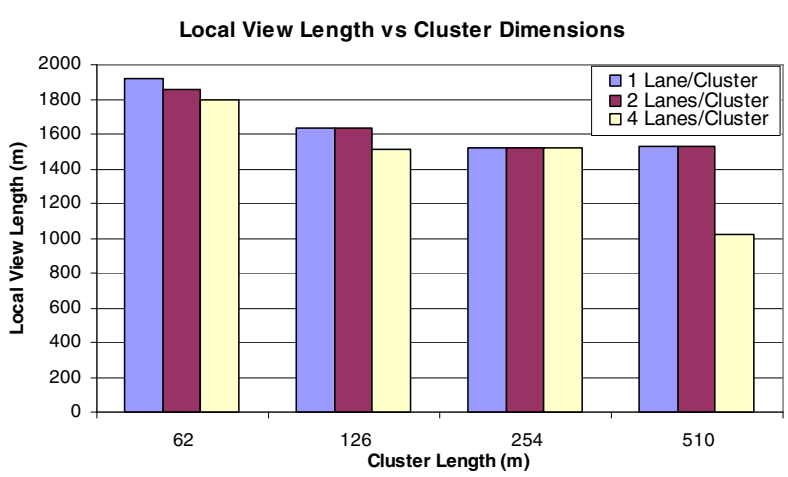

Figure 3. Local View Length as Cluster Dimensions Change

maximum number of clusters in the local view can be determined using Equation 12. As we are concerned with the maximum aggregated frame size, we calculate the size of the ACR in the case of having the maximum possible number of vehicles in each cluster, $V_{\max }$. Equation 13 shows how to determine the local view length, where $L C_{C}$ is the number of lanes per cluster $\left(L C_{C}=W_{C} / 4\right.$ since we assume that each lane is $4 \mathrm{~m}$ wide), and $L_{C}$ is the cluster length.

$$
\text { Length }=\frac{A C R \cdot \text { count }}{L C_{C}} * L_{C}
$$

In our analysis, we consider four different cluster lengths $(62 \mathrm{~m}, 126 \mathrm{~m}, 254 \mathrm{~m}$, and $510 \mathrm{~m})$ and three different cluster widths (1 lane, 2 lanes, and 4 lanes). The cluster lengths were chosen to maximize bit usage in the representation of $\Delta Y$ in the compact record. Using sign-magnitude representation and 6 bits for $\Delta Y$, we can represent positions in the range $[-31 \mathrm{~m}, 31 \mathrm{~m}]$ from the center of the cluster, for a total distance of $62 \mathrm{~m}$. In the same manner, a length of $126 \mathrm{~m}$ can be represented in just 7 bits for $\Delta Y, 254 \mathrm{~m}$ in 8 bits, and $510 \mathrm{~m}$ in 9 bits.

Figure 3 shows the local view length of each of the cluster dimensions. In Figure 3, the clusters with length $62 \mathrm{~m}$ have the longest local views. Within this group, the local view length decreases as the cluster width increases. For the clusters with length $126 \mathrm{~m}$, both the clusters with one lane width and two lanes width have the same local view length, while the cluster with four lanes width has a shorter local view. All the clusters with $254 \mathrm{~m}$ length have the same cluster length regardless of the cluster width. The last group, with cluster length $510 \mathrm{~m}$, behaves the same as the second group, where the clusters with one lane width and two lanes width have the same local view length, and the local view length for the cluster with four lanes width is the shortest of all the other cluster sizes. Since the cluster with $510 \mathrm{~m}$ length and four lanes width provides by far the shortest local view, we eliminate it from further analysis. 


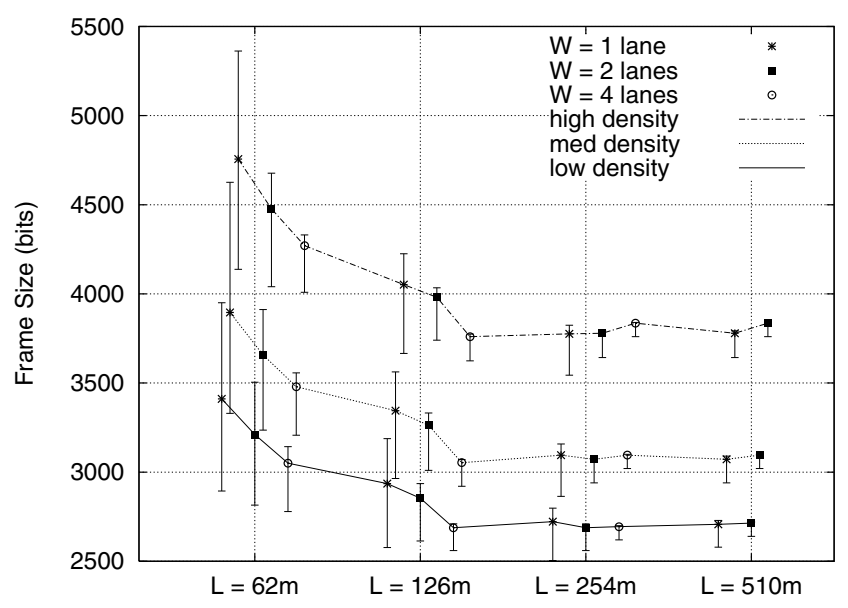

Figure 4. Minimum, Maximum, and Expected Aggregated Frame Sizes as Cluster Dimensions and Traffic Density Change

\subsection{Aggregated Frame Size Analysis}

The cluster dimensions are not the only factors that affect the size of the aggregated frame. Two other factors are the number of vehicles in the local view (ranging between 0 and $A C R$.count $* V_{\max }$ ) and how these vehicles are distributed over the local view clusters.

We calculate the aggregated frame size for various cluster sizes and also considering different traffic densities. We consider 53 vehicles $/ \mathrm{km}$ as low density, 66 vehicles $/ \mathrm{km}$ as medium density, and 90 vehicles/km as high density. For each traffic density, as the cluster dimensions change, the associated local view will change, which implies that the total number of vehicles in the local view, $N$, will change as well, as shown in Table 1 . In the table, $M$ is the number of clusters in the local view, and $K$ is the maximum number of vehicles per cluster. We distribute the vehicles over both the worst-case distribution to find the maximum frame size and the best-case distribution to find the minimum frame size. Figure 4 shows the minimum, maximum, and expected value of the aggregated frame size over different cluster sizes and traffic densities. (The calculation of the expected value of the aggregated frame size is given in the Appendix.) Each vertical line represents the possible frame sizes for the specific cluster dimension. The lowest point on the line is the minimum frame size, the highest point on the line is the maximum frame size, and the symbol in between is the expected value of the frame size. The expected value points for each traffic density are connected by a line to highlight the minimum expected frame size for that density. As explained in Section 3.2, the case of cluster length $510 \mathrm{~m}$ with a width of four lanes has been omitted.
From Figures 3 and 4, we find that a cluster width of 4 lanes and length of $126 \mathrm{~m}$ provides a small frame size along with long local view over various traffic densities.

In order to check how the system should behave in the case of having a highway with more than 4 lanes, we calculated the local view length for a highway with 5 lanes. In this case, the local view length dropped drastically, as shown in Table 2, which implies that increasing the cluster width to more than 4 lanes will provide no benefit. As a final conclusion, having a cluster with width of 4 lanes and length of $126 \mathrm{~m}$ has proved to be the optimal among any other cluster dimensions in CASCADE.

\section{Conclusion and Future Work}

We have presented an analysis of the CASCADE (Cluster-based Accurate Syntactic Compression of Aggregated Data in VANETs) data aggregation technique. In our analysis, we determined that a cluster size $16 \mathrm{~m}$ wide and $126 \mathrm{~m}$ long would provide the best trade-off between frame size and local view length. Having such optimal cluster size will reduce the bandwidth consumption and provide better extended driver visibility.

In future work, we plan to take advantage of the vehicles travelling in the opposite direction and study how this will affect our current analysis.

\section{Acknowledgments}

This work was supported by the National Science Foundation under Grant CNS-0721586.

We thank Hady Abdel-Salam and Dr. Stephan Olariu for assistance with calculating the expected value of the aggregated frame size.

\section{References}

[1] A. Bachir and A. Benslimane. A multicast protocol in ad hoc networks: Inter-vehicle geocast. In Proceedings of the IEEE Vehicular Technology Conference - Spring, pages 2456-2460, Jeju Island, Korea, Apr. 2003.

[2] K. Ibrahim and M. C. Weigle. Accurate data aggregation for VANETs (poster). In Proceedings of the ACM International Workshop on Vehicular Ad Hoc Networks (VANET), pages 71-72, Montreal, Canada, Sept. 2007.

[3] K. Ibrahim and M. C. Weigle. CASCADE: Cluster-based accurate syntactic compression of aggregated data in VANETs. Technical Report, available at http://www.cs.odu. edu/vanet/papers/ibrahim-TR-2008-02.pdf, Mar. 2008.

[4] C. Lochert, B. Scheuermann, and M. Mauve. Probabilistic aggregation for data dissemination in VANETs. In Proceedings of the ACM Workshop on Vehicular Ad Hoc Networks (VANET), pages 1-8, Montreal, Canada, Sept. 2007. 


\begin{tabular}{|c|c|c|c|c|c|c|c|}
\hline \multicolumn{2}{|c|}{ Cluster } & \multicolumn{3}{|c|}{ Local View } & Low & Medium & High \\
\hline $\begin{array}{l}\text { Length } \\
\text { (m) }\end{array}$ & $\begin{array}{l}\text { Width } \\
\text { (lanes) }\end{array}$ & $\begin{array}{l}\text { Length } \\
\text { (m) }\end{array}$ & M & K & $\begin{array}{c}\text { Density } \\
\mathrm{N}\end{array}$ & $\begin{array}{c}\text { Density } \\
\mathrm{N}\end{array}$ & $\begin{array}{c}\text { Density } \\
\mathrm{N}\end{array}$ \\
\hline$\overline{662}$ & 1 & 1922 & $\overline{124}$ & $\overline{\overline{8}}$ & $\overline{101}$ & $\overline{126}$ & $\overline{172}$ \\
\hline 62 & 2 & 1860 & 60 & 16 & 98 & 122 & 167 \\
\hline 62 & 4 & 1798 & 26 & 32 & 95 & 118 & 161 \\
\hline 126 & 1 & 1638 & 52 & 18 & 86 & 108 & 147 \\
\hline 126 & 2 & 1638 & 26 & 36 & 86 & 108 & 147 \\
\hline 126 & 4 & 1512 & 12 & 72 & 80 & 99 & 136 \\
\hline 254 & 1 & 1524 & 24 & 36 & 80 & 100 & 137 \\
\hline 254 & 2 & 1524 & 12 & 72 & 80 & 100 & 137 \\
\hline 254 & 4 & 1524 & 6 & 144 & 80 & 100 & 137 \\
\hline 510 & 1 & 1530 & 12 & 72 & 81 & 100 & 137 \\
\hline 510 & 2 & 1530 & 6 & 144 & 81 & 100 & 137 \\
\hline 510 & 4 & 1020 & 2 & 288 & 54 & 67 & 91 \\
\hline
\end{tabular}

Table 1. Data for All Cluster Dimensions

\begin{tabular}{|c|c|c|}
\hline \multicolumn{2}{|c|}{ Cluster } & Local View \\
$L_{C}$ & $\begin{array}{c}W_{C} \\
(\mathrm{~m})\end{array}$ & $\begin{array}{c}\text { Length } \\
(\mathrm{m})\end{array}$ \\
\hline \hline 62 & 4 & 1798 \\
62 & 5 & 1426 \\
126 & 4 & 1512 \\
126 & 5 & 1260 \\
254 & 4 & 1524 \\
254 & 5 & 1016 \\
510 & 4 & 1020 \\
510 & 5 & 1020 \\
\hline
\end{tabular}

Table 2. Comparing Local View Lengths in Case of Cluster Width of 4 Lanes and 5 Lanes

[5] T. Nadeem, S. Dashtinezhad, C. Liao, and L. Iftode. TrafficView: Traffic Data Dissemination using Car-to-Car Communication. ACM Mobile Computing and Communications Review (MC2R), Special Issue on Mobile Data Management, 8(3):6-19, July 2004.

[6] F. Picconi, N. Ravi, M. Gruteser, and L. Iftode. Probabilistic Validation of Aggregated Data in Vehicular Ad-hoc Networks. In Proceedings of the ACM Workshop on Vehicular Ad Hoc Networks (VANET), pages 76-85, Los Angeles, CA, Sept. 2006.

\section{Appendix}

We present the calculation of the expected value of the aggregated frame size. Each frame size corresponds to a different distribution of vehicles over the local view clusters. Some of the vehicle distributions are more frequent than others, so they occur with higher probability. The problem of distributing the vehicles over the local view clusters is similar to the occupancy problem with limited capacity.

Let us assume that we have $M$ clusters, $N$ vehicles, and the maximum capacity for each cluster cannot exceed $K$ vehicles. The vehicles can be distributed over the clusters as in Equation 14.

$$
A_{i}=\left(k_{1}, k_{2}, \ldots, k_{r}\right)
$$

where $0 \leq k_{i} \leq K$ and $r \leq\left\lceil\frac{N}{K}\right\rceil$.

$A_{i}$ is the general form for the possible distributions in which the number of vehicles in the $i_{t h}$ cluster is exactly $k_{i}$ vehicles. The total number of all possible vehicle distributions over the clusters, assuming the clusters are distinguishable, can be calculated from Equation 15.

$$
\begin{aligned}
\operatorname{Count}(N, M, K)= & \sum_{i=0}^{\left\lfloor\frac{N}{K+1}\right\rfloor}(-1)^{i}\left(\begin{array}{c}
M \\
i
\end{array}\right) \\
& \left(\begin{array}{c}
N+M-i(K+1)-1 \\
M-i
\end{array}\right)
\end{aligned}
$$

Each possible distribution $A_{i}$ will have an associated frame size, but it is possible for a frame size to be associated with more than one distribution. To calculate the expected frame size, we have to calculate the probability of each frame size, thus we have to count all possible distributions that can generate this frame size. If we study Equation 11 from Section 3.1.3 carefully, we notice that the frame size depends on the number of vehicles $N$, the number of clusters $M$, and the number of empty clusters. As $M$ and $N$ are constant for each possible cluster dimension, the only factor that will affect the frame size will be the number of empty clusters. The frame size $F S_{j}$ is generated when having $j$ empty cluster(s). The count of all possible distributions that can generate the frame size $F S_{j}$ can be calculated using Equation 15, after modifying the parameters $M, N$, and $K$ to be $M^{\prime}, N^{\prime}$, and $K^{\prime}$ as in Equation 16.

$$
\begin{aligned}
\operatorname{Count}_{j}\left(N^{\prime}, M^{\prime}, K^{\prime}\right)= & \sum_{i=0}^{\left\lfloor\frac{N^{\prime}}{K^{\prime}+1}\right\rfloor}(-1)^{i}\left(\begin{array}{c}
M^{\prime} \\
i
\end{array}\right) \\
& \left(\begin{array}{c}
N^{\prime}+M^{\prime}-i\left(K^{\prime}+1\right)-1 \\
M^{\prime}-i
\end{array}\right)
\end{aligned}
$$

where $\mathrm{j}$ is the number of empty clusters $0 \leq j \leq M-\left\lceil\frac{N}{K}\right\rceil$, $M^{\prime}=M-j, N^{\prime}=N-M^{\prime}$, and $K^{\prime}=K-1$.

Using Equation 15 and 16 we can calculate the probability of having frame size $F S_{j}$, as in Equation 17.

$$
\operatorname{Pr}\left(F S_{j}\right)=\frac{\operatorname{Count}_{j}\left(N^{\prime}, M^{\prime}, K^{\prime}\right)}{\operatorname{Count}(N, M, K)}
$$

Hence, the expected frame size will be calculated using Equation 18.

$$
E(F S)=\sum_{j=0}^{M-\left\lceil\frac{N}{K}\right\rceil} \operatorname{Pr}\left(F S_{j}\right) * F S_{j}
$$

Using the previous equations, we can calculate the expected frame size for the possible cluster dimensions with different traffic densities. 